\title{
Characterization of Living Anion Chain End of Oligomeric 1-Phenyl-1,3-butadienyllithium
}

\author{
Toshimitsu Suzuki, Yasushi TsujI, Yoshihisa WATANabe, \\ and Yoshinobu TAKEGAMI \\ Department of Hydrocarbon Chemistry, Faculty of Engineering, \\ Kyoto University, Kyoto, 606 Japan. \\ (Received February 16, 1979)
}

\begin{abstract}
MHz}{ }^{13} \mathrm{C}$ and $220 \mathrm{MHz}{ }^{1} \mathrm{H}$ NMR investigations were carried out on living anion chain ends of oligomeric 1-phenyl-1,3-butadiene (1PB) in order to reveal the anionic polymerization mechanism. The propagating species of $1 \mathrm{~PB}$ can be concluded to be the 4,1 anion. Lithiated 1-phenyl-2-butene (1P2B) was employed as a model anion of the propagating species of 1PB. A negative charge on the chain end delocalizes at $\alpha$-carbon and phenyl ring, and delocalization to $\gamma$-carbon is small regardless of the polymerization solvent. Therefore, the monomer attacks $\alpha$ carbon and consequently the in-chain 1,4 unit is predominant even in THF solvent. These behaviors are quite different from those observed in oligomeric butadiene and/or isoprene chain ends. In THF the living chain end is regarded as only trans-4,1, whereas in hydrocarbon media it is an almost equal mixture of trans- and cis-4,1 structures.

KEY WORDS 1-Phenylbutadiene / Anionic Polymerization / Living Oligomer $/{ }^{13} \mathrm{C}$ NMR / ${ }^{1} \mathrm{H}$ NMR / 1-Phenyl-2-butene / Metalation / Charge Distribution / Polymerization Mechanism /
\end{abstract}

We have recently elucidated microstructures of poly(1-phenyl-1,3- butadiene) [poly(1PB)] and poly(2-phenyl-1,3-butadiene) [poly(2PB)] prepared by anionic initiators. ${ }^{1,2}$ These results lead to the conclusion that $1 \mathrm{~PB}$ and $2 \mathrm{~PB}$ behave in quite different ways from butadiene (B) and isoprene (I) in anionic polymerization.

A large number of investigations have been carried out on the adducts of alkyllithium and B or I, using NMR spectroscopic results to account for the polymerization mechanism. ${ }^{3-14}$ These results clearly revealed the structures of the living chain ends and suggested the mechanism of the polymerizations. Recently Bywater et al. have studied benzylic alkali metal compounds by ${ }^{13} \mathrm{C}$ NMR spectroscopy in order to discuss the charge distribution of benzylic anions corresponding to the propagating species of styrene derivatives in anionic polymerization. ${ }^{15} \mathrm{On}$ the other hand, several NMR studies have been reported on phenyl allyl anions. ${ }^{16,17}$ Living anion chain ends of polymeric and oligomeric 1PB are considered to be quite similar to the phenyl allyl anion. ${ }^{18}$
In this study we have investigated ${ }^{13} \mathrm{C}$ and ${ }^{1} \mathrm{H}$ NMR spectra of living anions of oligomeric $1 \mathrm{~PB}$ under various conditions. It is of interest to see how the charge distributes in the anion chain end (covalent or ionic) and how the microstructure of poly(1PB) is controlled in a homogeneous polymerization system.

\section{EXPERIMENTAL}

\section{Materials}

trans-1-Phenyl-1,3-butadiene (1PB) was synthesized by the method described in a previous paper ${ }^{1}$. 1-Phenyl-2-butene (1P2B) was obtained from Tokyo Kasei Co. and purified by distillation over $\mathrm{LiAlH}_{4}$. The 1P2B thus obtained was the pure trans isomer, according to its IR and ${ }^{13} \mathrm{C}$ NMR spectra. $t$-BuLi was prepared from $t$-butyl chloride and lithium dispersion (Alfa) in pentane by the method reported by Smith. ${ }^{19} t$ - BuLi was used as benzene- $d_{6}$ solution. $t$-BuLi benzene- $d_{6}$ solution was prepared by displacing pentane with benzene- $d_{6}$ under high vacuum. 
Solvents were carefully purified by distillation in the presence of suitable drying reagents.

\section{Oligomerization and Metalation Procedure}

Oligomerization of $1 \mathrm{~PB}$ and metalation of 1P2B were carried out in NMR sample tubes in situ under a pure argon atmosphere with $t$ - BuLi at $5^{\circ} \mathrm{C}$. Concentrations of the anion chain end of oligomeric $1 \mathrm{~PB}$ were $0.06-0.30 \mathrm{~mol} \mathrm{dm}^{-3}$.

\section{${ }^{1} H$ and ${ }^{13} C$ NMR Spectra}

${ }^{1} \mathrm{H}$ NMR spectra were recorded on a Varian HR $220(220 \mathrm{MHz})$, and ${ }^{13} \mathrm{C}$ NMR spectra were measured with a JEOL JNM FX 100 spectrometer (25.0 $\mathrm{MHz}$ ). In some cases THF was used in the place of THF- $d_{8}$ to eliminate the appearance of multiplet solvent peaks due to ${ }^{13} \mathrm{C}-\mathrm{D}$ coupling. Very strong absorptions of solvent peaks compared with sample signals were carefully recorded within the dynamic range of the AD converter of the istrument (12 bits). Typical conditions for the ${ }^{13} \mathrm{C}$ measurements were: spectral width, $5 \mathrm{kHz}$; acquisition time, $0.812 \mathrm{~s}$; data points, 8192 ; pulse width, $7 \mu \mathrm{s}\left(42^{\circ}\right)$; pulse repetion, 2.0 s; number of transients $(80 \sim 128) \times 12$.

\section{RESULTS AND DISCUSSION}

\section{Propagating Species in Anionic Polymerization of $1 P B$}

The microstructure of poly(1PB) reported in a previous paper ${ }^{1}$ is summarized in Table I. Poly(1PB) has predominantly a trans-1, 4 structure even if the polymerization was carried out in THF. Cis-1,4 and 3,4 units increase with a decrease in the polarity of the polymerization solvent. This behavior is quite different from that of $B$ and $I$ in the anionic polymerization. In the polymerization of those monomers the presence of polar solvents increases the vinyl unit drastically. ${ }^{20}$ The origin of this difference in the behavior will be traced later.

The propagating species of 1PB in the anionic polymerization can be concluded to be the 4,1 anion (I), not the 1,4 anion (II), since the resulting polymers

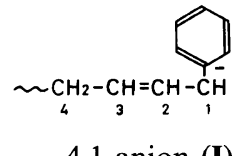

4,1 anion $(\mathbf{I})$

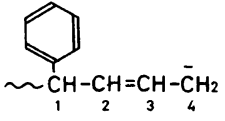

1,4 anion (II) contain only 1,4 and 3,4 units. The presence of the 4,1 propagating anion is supported by the result that the one to one adduct of $t$-BuLi and 1PB terminated with methanol consits of trans-4,1 cis-4,1, and 4,3 adducts as shown below.

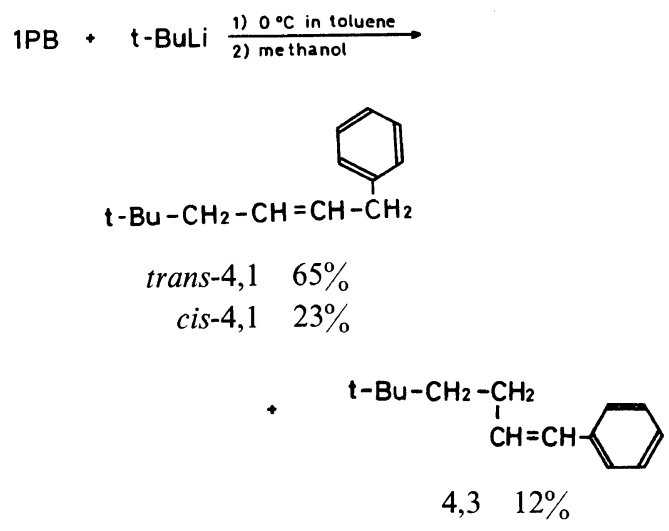

The product distribution of the one to one adduct was estimated by gas chromatography and their sum was normalized to $100 \%$. The amount of one to one adduct in the oligomeric species can be estimated as $65 \%$ of all the adducts from gel-permeation

Table I. Microstructure of poly(1-phenylbutadiene $)^{\mathrm{a}}$

\begin{tabular}{|c|c|c|c|c|c|c|c|c|}
\hline \multirow{2}{*}{ No. } & \multirow{2}{*}{ Initiator } & \multirow{2}{*}{ Solvent } & \multicolumn{2}{|c|}{ Polymerization } & \multirow{2}{*}{$\frac{\text { Conversion }}{\%}$} & \multicolumn{3}{|c|}{ Structure $/ \%$} \\
\hline & & & Temp $/{ }^{\circ} \mathrm{C}$ & Time $/ \mathrm{h}$ & & trans $-1,4$ & cis- 1,4 & 3,4 \\
\hline 13 & $t-\mathrm{BuLi}$ & THF & -78 & 4.0 & 100 & 79 & 11 & 10 \\
\hline 12 & $t-\mathrm{BuLi}$ & Benzene & 20 & 3.5 & 100 & 66 & 26 & 8 \\
\hline 11 & $t-\mathrm{BuLi}$ & Toluene & 20 & 3.5 & 100 & 56 & 28 & 16 \\
\hline 14 & $t-\mathrm{BuLi}$ & Hexane & 20 & 4.0 & 95 & 49 & 28 & 23 \\
\hline
\end{tabular}

a This is a summary of Table II in the previous paper (ref 1). 
chromatography.

\section{Characterization of Metalated $1 P 2 B$}

As it is difficult to obtain a pure $n$ ' mer chain end, a model compound of the chain end was employed for the NMR investigations. The model anion for the 4,1 propagating anion in the polymerization of $1 \mathrm{~PB}$ is prepared by metalating 1P2B with $t-\mathrm{BuLi}$ as shown in Scheme I. The reaction did not proceed in pure hydrocarbon solvent.

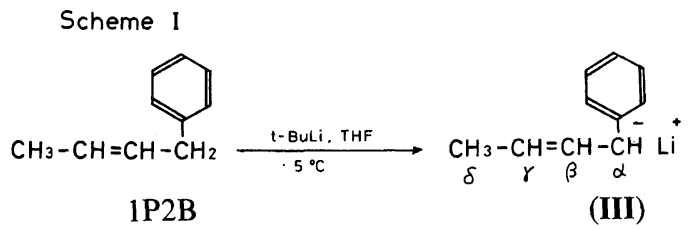

Figure 1 shows the $25.0 \mathrm{MHz}{ }^{13} \mathrm{C}$ NMR spectra of lithiated 1P2B (III) in THF. Chemical shifts of the carbon resonances are listed in Table II. Small resonances at 17.8 and $39.0 \mathrm{ppm}$ show the presence of a small quantity of unreacted $1 \mathrm{P} 2 \mathrm{~B} \cdot{ }^{13} \mathrm{C}$ chemical shifts and ${ }^{13} \mathrm{C}-\mathrm{H}$ coupling constants of phenylallyllithium compounds were first reported by van Dongen et al. ${ }^{16}$ The assignments were carried out by comparing the observed spectra with those reported in the literature, with the aid of the off-resonance spectra. In Figure 1 ortho and para carbon resonances of the phenyl ring appear at higher field than

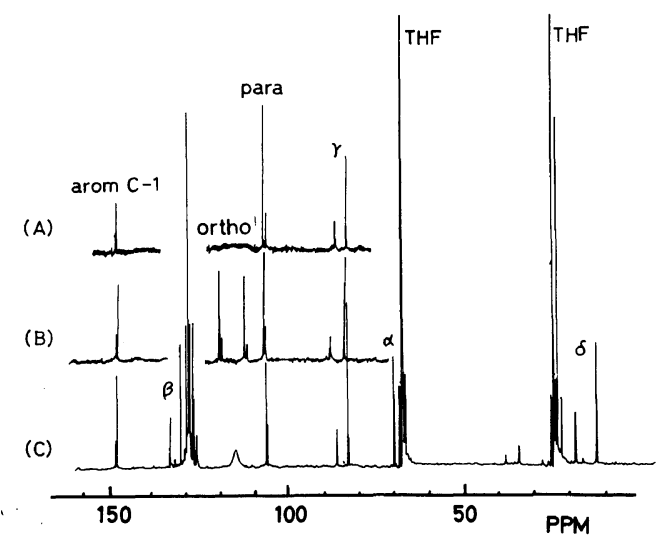

Figure 1. $25.0 \mathrm{MHz}{ }^{13} \mathrm{C} \mathrm{NMR}$ spectra of lithiated $1 \mathrm{P} 2 \mathrm{~B}$ (III) at various temperatures: (A) $0^{\circ} \mathrm{C}$, (B) $-45^{\circ} \mathrm{C}$, and (C) $24^{\circ} \mathrm{C}$; 1P2B $2.37 \mathrm{mmol}$, THF $1.5 \mathrm{~cm}^{3}$, and 3.00 mol dm ${ }^{-3} t$-BuLi benzene- $d_{6}$ solution, $0.87 \mathrm{~cm}^{3}$. ordinary phenyl carbon resonances such as toluene. The high-field shift of the para carbon resonance is particuarly noteworthy. This result indicates that the negative charge delocalizes to the phenyl ring and that the electron density increases especially at para position. The result is almost identical to those reported in phenylallyllithium, ${ }^{17}$ 1,3,3-trimethyl-1phenylbutyllithium ${ }^{21}$, 1,1-diphenylhexyllithium, ${ }^{22}$ 1,1-diphenylbutyllithium, ${ }^{23}$ and 1,3-diphenylallyllithium. ${ }^{24}$ At $24^{\circ} \mathrm{C}$ the ortho carbon resonance at $115.0 \mathrm{ppm}$ is much broader than the other resonances. Upon cooling, the broader resonance collapses at $0^{\circ} \mathrm{C}$, then splits into two sets of peaks (111.4 and $118.6 \mathrm{ppm})$ at $-45^{\circ} \mathrm{C}$. This phenomenon is explained by the presence of the rotational barrier of the $\alpha$-carbon-phenyl partial double bond. At $-45^{\circ} \mathrm{C}$ the rotation about this $\alpha$-carbon-phenyl bond is slow enough to distinguish two ortho carbons which are magnetically non-equivalent. By simulating the ortho carbon resonance at various temperatures using the equation proposed, ${ }^{25}$ the activation parameters $\Delta H^{\neq}=9.5 \mathrm{kcal} \mathrm{mol}^{-1}\left(39.7 \mathrm{~kJ} \mathrm{~mol}^{-1}\right)$ and $\Delta S^{\neq}=-12 \mathrm{cal} \mathrm{mol}^{-1} \mathrm{deg}^{-1}\left(-50.2 \mathrm{~J} \mathrm{~K}^{-1}\right.$ $\mathrm{mol}^{-1}$ ) could be obtained for the rotation.

Bywater et al. ${ }^{14}$ and Glaze et al. ${ }^{3,5}$ studied 5,5dimethyl-2-hexenyllithium (IV), produced by 1,4 addition of $t$ - BuLi and $\mathrm{B}$, using ${ }^{1} \mathrm{H}$ and ${ }^{13} \mathrm{C}$ NMR spectrometry. The ${ }^{13} \mathrm{C}$ and ${ }^{1} \mathrm{H}$ NMR data of (IV) previously reported are summarized in Table III. As is well known, (IV) exists as covalent species ( $\sigma$-allyl) in hydrocarbon solvents. On the other hand, in the presence of a donating solvent, the lithium cation was strongly coordinated by the solvent, and charge would thus be transferred to the organic moiety of (IV): ionic species ( $\pi$-allyl). The increase in charge would be expected to concentrate on $\gamma$ - and $\alpha$-carbon atoms.

The $\alpha$-carbon resonance of lithiated 1P2B (III) appears at $70.2 \mathrm{ppm}$. This value is $40-50 \mathrm{ppm}$ lower field than that for the $\alpha$-carbon of (IV), indicating its substantial $s p^{2}$ hybridization. The same results were obtained in other phenyl alkali metal salts. ${ }^{15,17,21}$ The $\gamma$-carbon resonance of lithiated 1P2B (III) appears at lower field than that of $\pi$-allyl (IV), showing smaller delocalization of charge to the $\gamma$ carbon.

Many experimental and theoretical results indicate that the ${ }^{13} \mathrm{C}$ chemical shift is an effective measure of the hydridization and charge density on the observing carbon. O'Brien proposed eq 1 for 
Table II. ${ }^{13} \mathrm{C}$ chemical shift ${ }^{\mathrm{a}}$ and $\pi$-electron density for lithiated $1 \mathrm{P} 2 \mathrm{~B}$ and living oligomeric $1 \mathrm{PBLi}$

\begin{tabular}{|c|c|c|c|c|c|c|}
\hline \multirow{3}{*}{ Position } & & & \multicolumn{4}{|c|}{ Living oligomeric $1 \mathrm{PBLi}$} \\
\hline & \multicolumn{2}{|c|}{ Lithiated 1P2B } & \multicolumn{2}{|c|}{$\delta$ in $\mathrm{ppm}$} & \multicolumn{2}{|c|}{$\rho^{\mathrm{c}}$} \\
\hline & $\delta$ in ppm & $\rho^{\mathrm{c}}$ & In THF & In benzene $e^{e}$ & In THF & In benzene \\
\hline$\alpha$ & 70.2 & 1.40 & 68.0 & 69.0 & 1.42 & 1.41 \\
\hline$\beta$ & $\begin{array}{l}130.6^{\mathrm{b}} \\
133.5^{2}\end{array}$ & $\begin{array}{l}1.02 \\
1.00\end{array}$ & $-^{d}$ & - & - & - \\
\hline$\gamma$ & $\begin{array}{l}83.3 \\
86.4\end{array}$ & $\begin{array}{l}1.32 \\
1.30\end{array}$ & 89.8 & 95.5 & 1.28 & 1.24 \\
\hline$\delta$ & $\begin{array}{l}11.9 \\
18.5\end{array}$ & - & $-^{\mathrm{d}}$ & - & - & - \\
\hline arom. $\mathrm{C}-1$ & $\begin{array}{l}148.4 \\
148.7\end{array}$ & $\begin{array}{l}0.90 \\
0.90\end{array}$ & 148.8 & 147.7 & 0.90 & 0.91 \\
\hline ortho & 115.0 & 1.12 & 115.6 & 114.3 & 1.11 & 1.12 \\
\hline meta & 128.5 & 1.03 & - & - & - & - \\
\hline para & $\begin{array}{l}106.7 \\
106.4\end{array}$ & 1.17 & 107.4 & 106.5 & 1.17 & 1.17 \\
\hline
\end{tabular}

a Shifts are recorded in ppm downfield from TMS using the following solvent peaks as standards: $\mathrm{C}_{6} \mathrm{D}_{6} 128.4$ and THF 67.0 .

b A set of data shows cis isomer aboye, and trans isomer below.

c $\pi$-electron density calculated with eq 1 .

d Cannot be identified as distinct resonances.

e In benzene- $d_{6}$.

the linear ${ }^{13} \mathrm{C}$ shift $(\delta)-\pi$-electron density $(\rho)$ relationship. ${ }^{26}$

$$
\delta=289.5-156.3 \rho
$$

$\pi$-Electron densities estimated from the ${ }^{13} \mathrm{C}$ chemical shift using eq 1 are listed in Table II.

The resonances of $\beta, \gamma, \delta$, arom.C-1, ortho (only at $-45^{\circ} \mathrm{C}$ ), and para carbons consist of two sets of peaks. Generally a carbon flanked by a cis olefin double bond appears at higher field than that flanked by a trans one. ${ }^{27}$ Therefore, juding from the intensities of the two resonances the predominant isomer would be the cis isomer. In the ${ }^{1} \mathrm{H}$ NMR spectrum (Figure 2), as will be discussed later, the $\delta$ proton resonance appears as two sets of doublet. This shows lithiated 1P2B (III) contains cis and trans isomers in the ratio of $3: 1 .^{* 1}$ Although the meta-

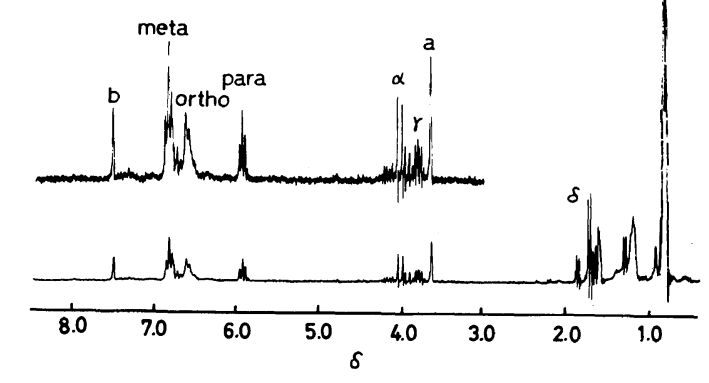

Figure 2. $220 \mathrm{MHz}{ }^{1} \mathrm{H}$ NMR spectrum of lithiated 1P2B (III): 1P2B $0.08 \mathrm{mmol}$, THF- $d_{8} 0.3 \mathrm{~cm}^{3}$, and $1.36 \mathrm{~mol} \mathrm{dm}^{-3} t$-BuLi benzene- $d_{6}$ solution, $0.15 \mathrm{~cm}^{3}$.

*1 H. Kloosterziel reported a cis-4,1 tendency for a potassium salt of $1 \mathrm{P} 2 \mathrm{~B}$ in ref 28 . 
lation was carried out with pure trans-1P2B, the cis isomer was obtained. This indicates the configuration of $\beta-\gamma$ bond is isomerized through a 4,3 anion as shown in Scheme II. In addition to cis-1P2B and trans-1P2B, 1-phenyl-1-butene was identified after protonation of lithiated 1P2B (III) with męthanol by gas chromatography. The ratio of cis-1P2B, trans1P2B, and 1-phenyl-1-butene was $35: 19: 46{ }^{* 2}$ It is surprising that 1-phenyl-1-butene was obtained as a main isomer after the protonation of the anion. However, no carbon resonances assignable to the 4,3 anion could be observed in Figure 1. The resonance effect of the phenyl ring will lead to the 4,1 anion. In the case of living oligomeric B and I, neither 1,2 nor 4,3 anion chain ends could be detected, even in THF medium, by NMR spectroscopies. ${ }^{20}$

Figure 2 shows the $220 \mathrm{MHz}^{1} \mathrm{H}$ NMR spectra of lithiated 1P2B (III). The spectra show the same features as were observed in ${ }^{13} \mathrm{C}$ NMR spectra. Phenyl proton resonances show a high-field shift. $\alpha$ Proton resonances appear as a doublet at considerably lower field ( $\delta 4.1)$ as compared with the $\alpha$ proton resonance of (IV). The changes in chemical shifts indicate a substantial amount of charge delocalization into the phenyl ring and the presence of a partial double bond between the $\alpha$-carbon and phenyl ring. The $\gamma$-proton resonance of lithiated 1P2B (III) appears at $\delta 3.8-4.2$ as a complicated multiplet. This value is almost midway between $\sigma$ and $\pi$-allyl (IV) in cis isomer. ${ }^{* 3}$ It is difficult to compare the chemical shift of lithiated 1P2B (III) with that of (IV). However, it is safe to say that the delocalization of the negative charge to $\gamma$-carbon in lithiated 1P2B (III) is smaller than in $\pi$-allyl (IV). The

*2 Although this result is inconsistent with the microstructure of poly(1PB) obtained in THF, the product distributions after protonation of phenylallyl anions are quite sensitive to the structures of organic moieties. ${ }^{29,30}$ Lithiated 1P2B, therefore, cannot strictly be a model compound of the propagating chain end of $1 \mathrm{~PB}$. However, comparing the results obtained here and those in the literature, ${ }^{16}$ the ${ }^{13} \mathrm{C}$ chemical-shift data obtained for lithiated $1 \mathrm{P} 2 \mathrm{~B}$ can be safely used to understand the ${ }^{13} \mathrm{C}$ NMR spectra of the living oligomeric $1 \mathrm{~PB}$. The larger amount of 1-phenyl-1-butene is suggested by the higher-field shift of $\gamma$ proton and carbon resonances of the lithiated 1P2B, compared with those of the oligomeric 1PB anion.

*3 Lithiated $1 \mathrm{P} 2 \mathrm{~B}(\mathbf{I I I})$ contains predominantly cis isomer. Therefore, we adopt the results of $c i s-(I V)$ for the comparison.

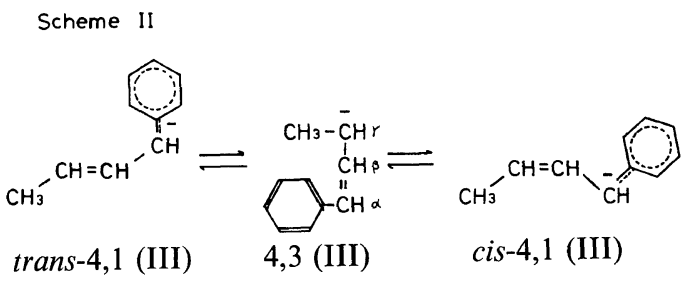

negative charge of lithiated 1P2B (III) must delocalize at $\alpha$-carbons and phenyl rings and the delocalization to $\gamma$-carbon is small.

\section{Characterization of Living Chain End of Oligomeric $1 P B$}

Unlike the metalation of 1P2B, the oligomerization of $1 \mathrm{~PB}$ can be carried out in pure hydrocarbon solvents. Figure 3 shows the ${ }^{13} \mathrm{C}$ NMR spectra of the living oligomeric 1PB. Although resonances are broader and more complicated due to in-chain units, they are essentially the same as that of

(Aa)

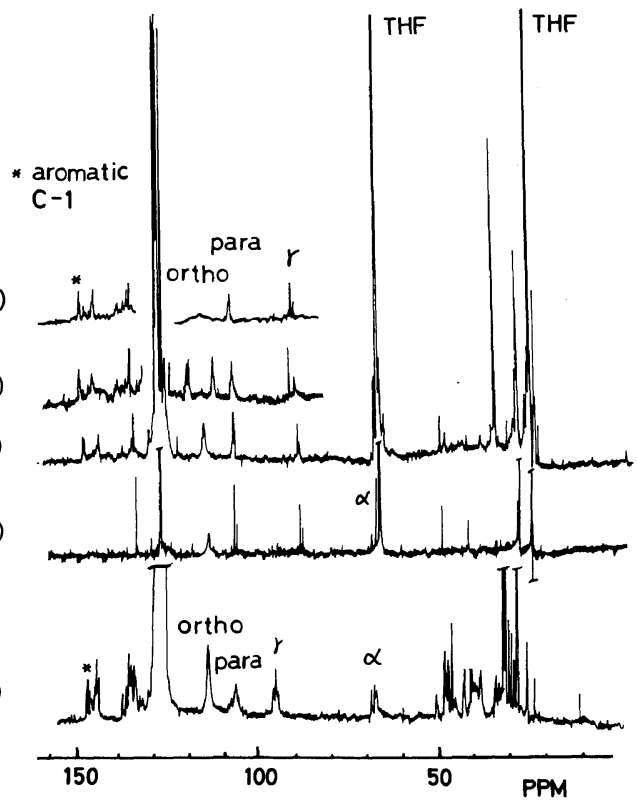

Figure 3. $25.0 \mathrm{MHz}{ }^{13} \mathrm{C} \mathrm{NMR}$ spectra of living oligomeric $1 \mathrm{~PB}$ at various temperatures: (Aa) $6^{\circ} \mathrm{C}$, (Ab) $-40^{\circ} \mathrm{C}$, and (Ac) $24^{\circ} \mathrm{C}$ in $\mathrm{THF}$; (Ad) spectrum recorded at $24^{\circ} \mathrm{C}$ by applying a $180^{\circ}-5.0 \mathrm{~s}-90^{\circ}-20 \mathrm{~s}$ pulse sequence; (B) $24^{\circ} \mathrm{C}$ in benzene- $d_{6} .(\mathrm{Aa}-\mathrm{c}) \overline{\mathrm{DP}}=5.8,(\mathrm{Ad}), \overline{\mathrm{DP}}=3.6$, and (B) $\overline{\mathrm{DP}}=6.0$. 
lithiated 1P2B(III) in Figure 1. Chemical shifts of the resonances and $\pi$-electron densities estimated by eq 1 are listed in Table II. The spectrum shows a highfield shift for the ortho and para carbons, and a down-field shift for the aromatic C-1 carbon, as compared with the corresponding shifts of 1P2B. Similar chemical-shift trends were seen for lithiated 1P2B (III) The changes in chemical shifts of the aromatic carbons indicate a substantial amount of charge delocalization into the phenyl ring. As observed in the ${ }^{13} \mathrm{C}$ NMR spectra of lithiated 1P2B (III), the shape of the ortho carbon resonance depends on the sample temperature. At $24^{\circ} \mathrm{C}$ the ortho carbon resonance is a singlet. Upon cooling, the singlet collapses at $6^{\circ} \mathrm{C}$, then splits into two distinct peaks at $-40^{\circ} \mathrm{C}$. This is also explained by the decrease in the rotation of the $\alpha$-carbon-phenyl partial double bond. The activating parameters $\Delta H^{\neq}=7.7 \mathrm{kcal} \mathrm{mol}^{-1}\left(32.2 \mathrm{~kJ} \mathrm{~mol}^{-1}\right), \Delta S^{\neq}=-19$ cal $\mathrm{mol}^{-1} \mathrm{deg}^{-1}\left(-79.5 \mathrm{~J} \mathrm{~K}^{-1} \mathrm{~mol}^{-1}\right)$ are obtained for the rotation. The $\alpha$-carbon resonance appears at $69.0 \mathrm{ppm}$ in benzene- $d_{6}$ (Figure 3B). However, it seems to be overlapped with a strong solvent resonance in THF (Figure 3Ac). When a $180^{\circ}-5.0 \mathrm{~s}-90^{\circ}-20 \mathrm{~s}$ pulse sequence was employed to this sample, intensities of the solvent resonances decreased and the $\alpha$-carbon resonance appeared at $68.0 \mathrm{ppm}$, as shown in Figure 3Ad. These $\alpha$-carbons appear at considerably lower field than that of (IV). In addition, the $J_{13_{\mathrm{C}-1}{ }_{\mathrm{H}}}$ of the $\alpha$-carbon in benzene$d_{6}$ is $149 \mathrm{~Hz}$. The substantial $s p^{2}$ hybridization of the $\alpha$-carbon is established by these results. The $\gamma$-carbon resonances appear at lower field than that of $\pi$-allyl (IV), showing a limited distribution of the negative charge at $\gamma$ position. The $\gamma$-carbon resonance appears at a slightly higher field in THF than in benzene- $d_{6}$. The negative charge seems to delocalize to $\gamma$ position in THF more than in benzene- $d_{6}$. However, the difference is smaller than the difference observed between $\sigma$ - and $\pi$-allyl (IV) chain ends, which is estimated to be $21-24 \mathrm{ppm}$ according to Table III.

Figure 4 shows ${ }^{1} \mathrm{H}$ NMR spectra of the living

Table III. ${ }^{1} \mathrm{H}$ and ${ }^{13} \mathrm{C}$ chemical shift of 5,5-dimethylhexene-2-yllithium

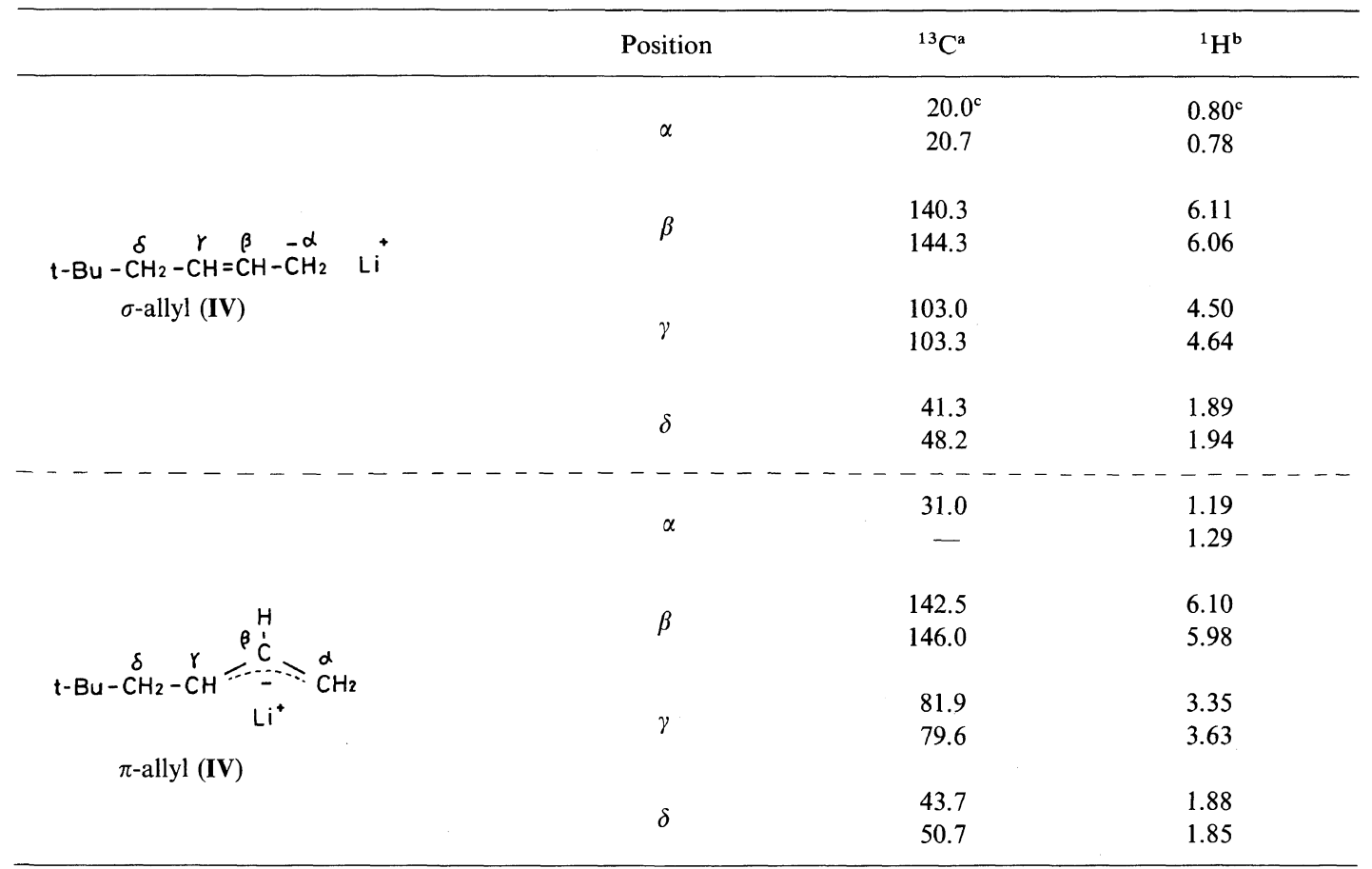

a Data in ref 14.

b $\delta$ from TMS; data in ref 3 and 5 .

c A set of data shows cis isomer above, and trans isomer below. 
(A)

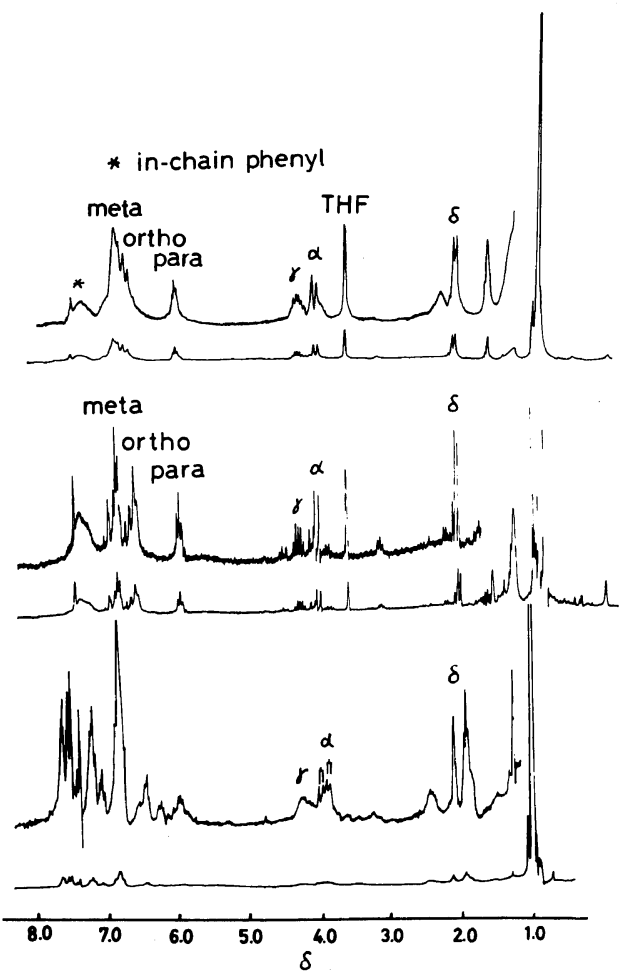

Figure 4. $220 \mathrm{MHz}{ }^{1} \mathrm{H}$ NMR spectra of living oligomeric $1 \mathrm{~PB}$ : (A) $\overline{\mathrm{DP}}=1.2$ in THF- $d_{8}$, (B) $\overline{\mathrm{DP}}=3.2$ in THF $-d_{8}$, and (C) $\overline{\mathrm{DP}}=3.5$ in toluene- $d_{8}$.

oligomeric 1PB. The higher-field shift of pheyl proton resonances and the lower-field shift of $\alpha$ proton resonance indicate the delocalization of charge to the phenyl ring and the presence of a partial double bond between the phenyl ring and the $\alpha$-carbon, which were suggested by the ${ }^{13} \mathrm{C}$ NMR spectra. A $\gamma$-proton of the oligomeric 1PB chain end appears at $\delta 4.3$ as a complicated multiplet in both
THF- $d_{8}$ and toluene- $d_{8}$, indicating that the nature of the solvent has almost no effect on the distribution of the negative charge. Furthermore, the chemical shift of the $\gamma$-proton is closer to that of $\sigma$-allyl (IV) than that of $\pi$-allyl (IV). This shows that the delocalization of the charge to $\gamma$-carbon is small regardless of the nature of a solvent. These results are quite different from those observed in oligomeric B and I chain ends. In those cases the distribution of charge is strongly affected by the nature of the solvent. In hydrocarbon media the chain end is reported to be $\sigma$ allyl (covalent), in which the charge localizes at $\alpha$ carbon; this chain end is believed to form an in-chain 1,4 unit when the next monomer adds to it. But, in polar media such as THF the chain end is regarded as $\pi$-allyl (ionic) where the charge delocalizes from $\alpha$ to $\gamma$-carbon, and this chain end is considered to form an in-chain vinyl unit preferentially. ${ }^{17}$

The negative charge, when considered with the information as far obtained, distributes in the chain end of oligomeric 1PB as follows: (a) The negative charge delocalizes at the $\alpha$-carbon and the phenyl ring. (b) The $\alpha$-carbon is substantially $s p^{2}$ hybridized due to the delocalization. The lithium atom must be interacting with $\pi$-electrons. (c) The delocalization to the $\gamma$-carbon is small. (d) The distribution of the charge is hardly affected by the nature of the solvent. From these results we could describe the living chain end of the oligomeric 1PB as shown in $(\mathbf{V})$.

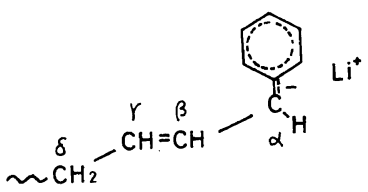

(V)

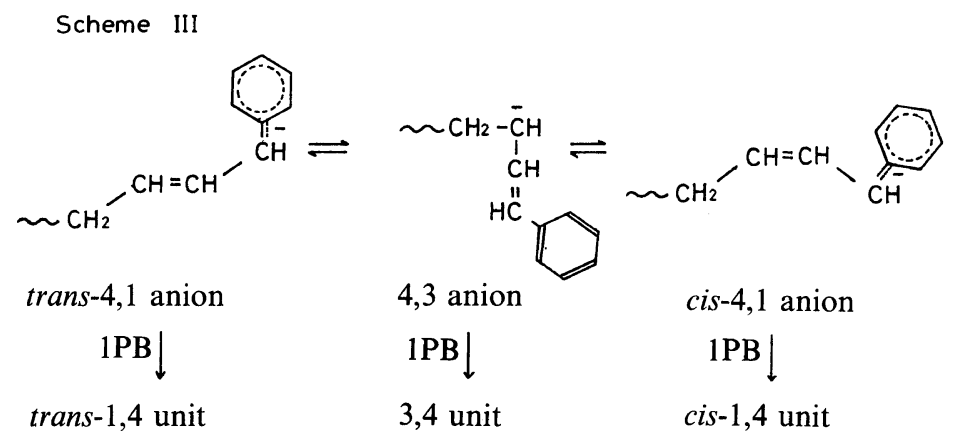



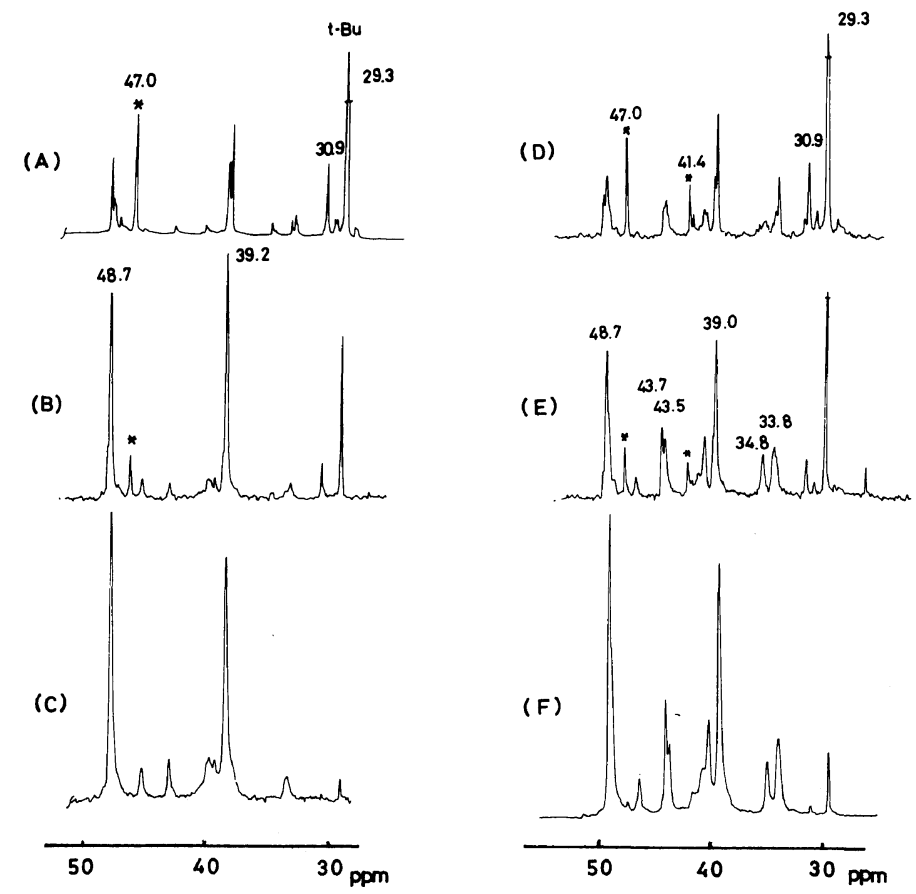

Figure 5. ${ }^{13} \mathrm{C}$ NMR spectra of methanol terminated oligomeric $1 \mathrm{~PB}:(\mathrm{A}) \mathrm{DP}=2.5,(\mathrm{~B}) \mathrm{DP}=20$, and $(\mathrm{C})$ $\overline{\mathrm{DP}}=90$ prepared in THF; (D) $\overline{\mathrm{DP}}=2.4$, (E) $\overline{\mathrm{DP}}=15$, and (F) $\overline{\mathrm{DP}}=75$ prepared in benzene. $*$ shows the terminal methylene carbon.

\section{Geometry of Double Bond in the Chain End of Oligomeric $1 P B$}

In Figure $4 C$ the $\alpha$-proton resonance consists of two sets of doublets in toluene- $d_{8}$, while it is a single doublet in THF- $d_{8}$ (Figure 4A, B). $\delta$-Proton resonance also appears at $\delta 1.9$ and $\delta 2.1$ in toluene- $d_{8}$, whereas it appears only at $\delta 2.1$ in THF- $d_{8}$. In hydrocarbon solvents trans- and cis-4,1 chain ends must coexist at an equilibrium through the 4,3 anion, as shown in Scheme III. The effect of association of the living end could not be established by means of NMR spectra. However, the initiation and propagation rates are much slower in hydrocarbon solvents than in THF. This must be due to the association of the alkyllithium and the living chain end. On the other hand, in THF- $d_{8}$ the living chain end seems to be preferentially trans-4,1. The effect of such a trans-cis isomerization on the microstructure of polyisoprene was recently investigated by Bywater et al. ${ }^{29}{ }^{13} \mathrm{C}$ NMR spectra of methanol terminated oligomeric 1PB clearly supported the above results. As shown in Figures 5A, B, and C, the methanol terminated oligomeric 1PB prepared in THF have only trans $-4,1$ chain ends $(47.0 \mathrm{ppm})$, which is the same as that of a model trans $-4,1$ end $(47.0 \mathrm{ppm})$. On the other hand, as shown in Figures 5D, E, and F, samples prepared in benzene have both trans-4,1 (47.0 ppm) and $c i s-4,1(41.4 \mathrm{ppm})$ chain ends (relative intensity is trans $/$ cis $\simeq 6 / 4^{* 4}$ ). The chemical shift of the $c i s-4,1$ chain end is close to that of a model cis-4,1 end (41.1 ppm). The model compounds, transand cis-1-phenyl-5,5-dimethyl-2-hexene, for the chain end of terminated oligomeric 1PB were fractionally collected from the adducts of $t$-BuLi and 1PB by gas chromatography on a column DEGA $2.25 \mathrm{~m}$. The ${ }^{13} \mathrm{C}$ chemical shifts of the model compounds are shown below.

*4 Cis-4,1 isomer was predominant in the case of lithiated 1P2B(III). The configuration of 1,4 double bonds seems to depend on the structure, as reported in ref 30 . 


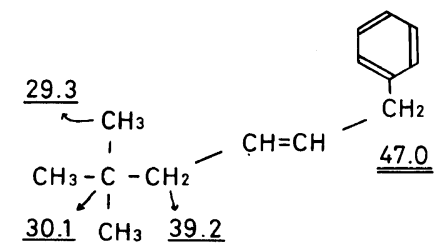

trans-1-phneyl-5,5-dimethyl-2-hexene; model for trans $-4,1$ chain end

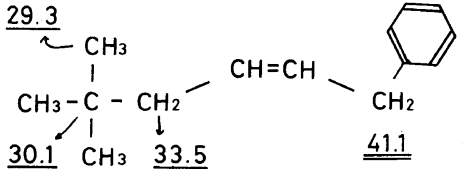

cis-1-phenyl-5,5-dimethyl-2-hexene; model for $c i s-4,1$ chain end

\section{Polymerization Mechanism of $1 P B$ in the Anionic Polymerization}

The behavior of $1 \mathrm{~PB}$ in the anionic polymerization is described as follows. Since the negative charge delocalizes at the $\alpha$-carbon and the phenyl ring, and the delocalization to $\gamma$-carbon is limited regardless of the polymerization solvent, the monomer adds to the $\alpha$-carbon, and consequently 1,4 units are predominant in in-chain units. Since the resonance effect of the phenyl ring on the stabilization of the anion is prevailing in the case, the distribution of the negative charge was hardly affected by the solvation of the lithium cation. ${ }^{* 5}$ The configuration of the in-chain 1,4 unit is determined by that of the anion 4,1 chain end. In THF the anion chain end is regarded as only trans-4,1, whereas in hydrocarbon media there is an almost equimolar mixture of trans- and cis-4,1 chain ends. They must be in equilibrium through 4,3 anions. By the addition of the monomer to the $c i s-4,1$ and 4,3 anion in the equilibrium, cis-1,4 and 3,4 units can increase in the polymers prepared in hydrocarbon media.

Acknowledgement. This work was supported in part by the Grant in Aid, Ministry of Education, Japan (No. 243019).

\footnotetext{
*5 trans-1-(4-methoxyphenyl)-1,3-butadiene was prepared with $p$-anisaldehyde and triphenylallylphosphonium bromide, followed by repeated recrystallizations from a hexane solution at $-30^{\circ} \mathrm{C}$, and polymerized with $t$-BuLi in THF. It was expected from the Hammett's substituent constant that the charge was transferred to $\gamma$-carbon from a phenyl ring due to the $p$-methoxy group in the propagating chain end and that the number of 3,4 units would increase in the resulting polymer. However, the microstructure of the polymer was exactly the same as that of poly(1PB) prepared under the same conditions. The electron donating power of the $p$-methoxy group might be too weak to affect the distribution of the negative charge in the chain end.
}

\section{REFERENCES}

1. T. Suzuki, Y. Tsuji, and Y. Takegami, Macromolecules, 11, 639 (1978).

2. T. Suzuki, Y. Tsuji, Y. Takegami, and H. J. Harwood, Macromolecures, 12, 234 (1979).

3. W. H. Glaze, J. E. Hanicak, M. L. Moore, and J. Chaudhuri, J. Organometal. Chem., 44, 39 (1972).

4. W. H. Glaze, J. E. Hanicak, D. J. Berry, and D. P. Duncan, J. Organometal. Chem., 44, 49 (1972).

5. W. H. Glaze, J. E. Hanicak, J. Chandhuri, M. L. Moore, and D. P. Duncan, J. Organometal. Chem., 51, 13 (1973).

6. J. Sledz, F. Schué, B. Kaempf, and S. Libs, Eur. Polym. J., 10, 1207 (1974).

7. S. Brownstein, S. Bywater, and D. J. Worsfold, Macromolecules, 6, 715 (1973).

8. S. Bywater, D. J. Worsfold, and G. Hollingsworth, Macromolecules, 5, 389 (1972).

9. F. Schué, D. J. Worsfold, and S. Bywater, Macromolecules, 3, 509 (1970).

10. M. Morton, R. D. Sanderson, and R. Sakata, J. Polym. Sci., Polym. Lett. Ed., 9, 61 (1971).

11. M. Morton, R. D. Sanderson, R. Sakata, and L. A. Falvo, Macromolecules, 6, 186 (1973).

12. M. Morton, R. D. Sanderson, and R. Sakata, Macromolecules, 6, 181 (1973).

13. R. Salle and J. Gole, J. Polym. Sci., Polym. Chem. Ed., 15, 1199 (1977).

14. S. Bywater and D. J. Worsfold, J. Organometal. Chem., 159, 229 (1978)

15. S. Bywater, P. Lachance, and D. J. Worsfold, J.'Phys. Chem., 79, 2148 (1975).

16. J. P. C. M. van Dongen, H. W. D. van Dijkman, and M. J. A. de Bie, Recl. Trav. Chim. Pays-Bas, 93, 29 (1974).

17. V. R. Sandel, S. V. McKinley, and H. H. Freedman, J. Am. Chem. Soc., 90, 495 (1968).

18. R. Asami and Y. Kondo, Preprint, The 31st Annual meeting of the Chemical Society of Japan, Tokyo, Autumn, 1974 2W07.

19. W. N. Smith, Jr., J. Organometal. Chem., 82, 1 (1974).

20. M. Morton and L. J. Fetters, Rubber Chem. Technol., 48, 359 (1975).

21. R. Wicke and K. F. Elgert, Makromol. Chem., 178, 
3063 (1977).

22. L. D. McKeever and R. Waack, J. Organometal., Chem., 28, 145, (1971).

23. Y. Okamoto and H. Yuki, J. Organometal. Chem., 32, 1 (1971).

24. H. H. Freedman, V. R. Sandel, and B. P. Thill, J. Am. Chem. Soc., 89, 1762 (1967).

25. F. A. Bovey, "Nuclear Magnetic Resonance Spectroscopy," Academic Press, New York N.Y. 1969, Chapter 7.
26. D. H. O'Brien, A. J. Hart, and C. R. Russell, J. Am. Chem. Soc., 97, 4410 (1975).

27. J. B. Stothers, "Carbon-13 NMR Spectroscopy," p 80, Academic Press, New York, N.Y., 1972.

28. H. Kloosterziel and J. A. A. van Drunen, Recl. Trav. Chim. Pays-Bas, 87, 1025 (1968).

29. D. J. Worsfold and S. Bywater, Macromolecules, 11, 582 (1978).

30. M. Schlosser and J. Hartmann, J. Am. Cem. Soc., 98, 4674 (1976). 